\title{
ON THE HOMOTOPY SET [BT, BG] WITH $\Pi$ FINITE AND G A COMPACT CONNECTED LIE GROUP
}

Journal Article

Author(s):

Mislin, G.; Thomas, C.

Publication date:

1989

Permanent link:

https://doi.org/10.3929/ethz-b-000423104

Rights / license:

In Copyright - Non-Commercial Use Permitted

Originally published in:

The Quarterly Journal of Mathematics 40(1), https://doi.org/10.1093/qmath/40.1.65 


\title{
ON THE HOMOTOPY SET $[B \pi, B G]$ WITH $\pi$ FINITE AND $G$ A COMPACT CONNECTED LIE GROUP
}

\author{
By G. MISLIN AND C. THOMAS
}

Dedicated to Ioan James on the occasion of his sixtieth birthday

[Received 28 October 1987]

LET $\pi$ be a finite group and $G$ equal $U(n), 0(n)$ or Sp $(n)$. Using the methods of $[\mathbf{A}]$ one can determine the image of the homotopy set $[B \pi, B G]$ in $K F(B \pi)$ for $F=\mathbb{C}, \mathbb{R}$ or $H$. In all three cases one finds that a homotopy class is stably represented by a virtual representation, and our aim in the present paper is to given some evidence for believing that a similar result holds without allowing $n$ to tend to infinity. Our starting points are the paper of $W$. Dwyer [D], which solves the problem for groups of prime power order and the determination by one of us of the homotopy set $[B S U(2), B S U(2)]$ by restriction to finite subgroups of $S U(2)$, see [M]. In part the latter argument succeeds because all finite subgroups of $S U(2)$ have period equal to 4 or 2 in cohomology, and in this paper we shall apply similar methods to determine $[B \pi, B G]$ for more general Lie groups $G$ at the price of restricting $\pi$ to be a group with arbitrarily large period in cohomology. Our results are most complete when $G=S U(2)$, and can be summarized in the diagram, in which $K$ stands for complex $K$-theory and $R$ for the complex representation ring:

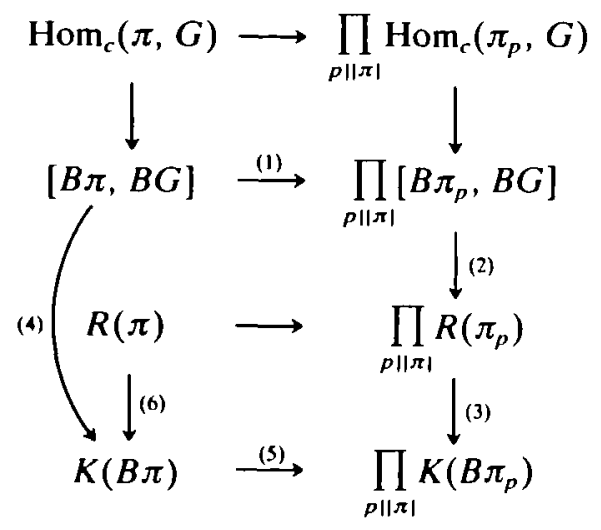

The horizontal maps are all induced by inclusion of subgroups, and our main result (Theorem 3.2 below) states that (1) is a monomorphism onto Quart. J. Math. Oxford (2), 40 (1989), 65-78 (C) 1989 Oxford University Press 0033-5606/89\$3.00 
the subset of stable classes. The top vertical arrow on the right is an isomorphism by Dwyer's work, which implies that (2) is a well-defined monomorphism. (3) is completion with respect to the augmentation ideal topology and is well known to be monic for $p$-groups, although not when $G$ has composite order (6). From [A] we know that the image of (4) is contained in the image of (6). That (5) is a monomorphism is a general property of cohomology theories. It follows that (4) is also $(1-1)$, justifying our claim that at least for certain groups Adams' results can be destablised: The homotopy classes $B \pi \rightarrow B G$ can be described by means of (equivalence classes) of virtual representations.

The interest of the second author in these ideas arises from his work on free actions by finite groups on $S^{3}$. It is still an open question whether there exist free non-linear actions by certain groups $Q(8 n ; k, l)$, which are extensions of $C_{k l}$ by the binary dihedral group $D_{8 n}^{*}$ of order $8 n$ ( $n, k, l)$ odd and mutually coprime). Since $Q$ is of Type II, see [WF] for the definition, Theorem 2.6 below holds and we can determine $[B Q, B$ Spin (4)]. Unfortunately it turns out that this homotopy set contains elements $f$ such that the Euler class $e(f)$ generates $H^{4}(Q, Z)$, thus providing candidates for the $k$-invariant of a free action. Hence the argument sketched in [T1] has a gap, which however can be closed by a geometric argument, at least when certain covering space assumptions are satisfied, see [T2].

This is intended to be the first in a series of papers. In the second we propose to explore the implications of the examples in Section 4 below for the structure of the Swan subgroup $T(Z \pi)$ of the projective class group $\tilde{K}_{0}(Z \pi)$. The idea is to use elements in $[B \pi, B U(n)]$ represented by the difference of two homorphisms to construct free actions on Brieskorn varieties (and their generalizations). At least if $\pi$ is metacyclic of odd order it is possible to replace the orbit space by a spherical space form, the $k$-invariant of which is closely related to the top dimensional Chern class of the original $U(n)$-bundle over $B \pi$. In further papers we propose as far as possible to extend the result in [M] to the homotopy set $[B S U(m), B S U(m)], m \geqslant 3$, starting from [I] calculations of J. Hubbuck and the cohomology of $p$-groups of low rank.

\section{The $p$-normalizer condition}

Let $\pi$ be a finite group and $\pi_{p}$ a $p$-Sylow subgroup of $\pi$. Recall that $\pi$ is called $p$-normal if the centre $c\left(\pi_{p}\right)$ of $\pi_{p}$ has the property that it equals the centre of any other $p$-Sylow subgroup which contains it, $[\mathbf{Z}]$.

LEMMA 1.1. If the finite group $\pi$ has periodic cohomology then $\pi$ is p-normal for all primes $p$. 
Proof. If $p$ is odd, $\pi_{p}$ is cyclic and so $\pi$ is $p$-normal. For $p=2$, either $\pi_{2}$ is cyclic and the same argument applies, or $\pi_{2}$ is a binary dihedral (generalised quaternion) group. In this case $c\left(\pi_{2}\right)$ is generated by the unique element of order 2 , which must be central in any 2-Sylow subgroup which contains it.

Let $N_{\pi}\left(\pi_{p}\right)$ denote the normalizer of $\pi_{p}$ in $\pi$, and $N_{\pi}\left(c\left(\pi_{p}\right)\right)$ the normalizer of its centre. When there is no risk of confusion we shall suppress the suffix $\pi$. Clearly $\pi_{p} \subseteq N\left(\pi_{p}\right) \subseteq N\left(c\left(\pi_{p}\right)\right)$.

DefinITION 1.2. The finite group $\pi$ satisfies the $p$-normalizer condition if $\pi$ is $p$-normal, and the inclusion $N\left(\pi_{p}\right) \subseteq N\left(c\left(\pi_{p}\right)\right)$ induces an isomorphism

$$
H_{*}\left(N\left(\pi_{p}\right) ; \mathrm{F}_{p}\right) \stackrel{\cong}{\rightrightarrows} H_{*}\left(N\left(c\left(\pi_{p}\right)\right) ; \mathbb{F}_{p}\right) .
$$

Using results of R. G. Swan, see [S], one proves

Lemma 1.3. Suppose that the finite group $\pi$ satisfies the $p$-normalizer condition. Then

$$
\operatorname{Res}_{\pi \rightarrow \pi_{p}}: H^{*}\left(\pi ; \mathbb{F}_{p}\right) \stackrel{\fallingdotseq}{\rightrightarrows} H^{*}\left(\pi_{p} ; \mathbb{F}_{p}\right)^{N\left(\pi_{p}\right)}
$$

is an isomorphism.

Proof. The superscript $N\left(\pi_{p}\right)$ refers to elements invariant under conjugation. By [S, Corollary to the Appendix] one has

$$
\operatorname{Res}_{\pi \rightarrow N\left(\pi_{p}\right)}: H^{*}\left(\pi ; \mathbb{F}_{p}\right) \stackrel{\rightrightarrows}{\rightrightarrows} H^{*}\left(N\left(\pi_{p}\right) ; \mathbb{F}_{p}\right) \text {. }
$$

Since $\pi_{p}$ is normal in $N\left(\pi_{p}\right)$ with index not divisible by $p$, the spectral sequence of a group extension shows that

$$
\operatorname{Res}_{N\left(\pi_{p}\right) \rightarrow \pi_{p}}: H^{*}\left(N\left(\pi_{p}\right) ; \mathbb{F}_{p}\right) \stackrel{\cong}{\rightrightarrows} H^{*}\left(\pi_{p} ; \mathbb{F}_{p}\right)^{N\left(\pi_{p}\right)} .
$$

Since restriction is natural the lemma follows.

The following are simple examples of groups satisfying the $p$ normalizer condition.

LeMMA 1.4. The finite group $\pi$ satisfies the p-normalizer condition if any of the following assumptions holds.

(i) $\pi_{p}$ is abelian

(ii) $\pi_{p}$ is normal in $\pi$

(iii) There is a split epimorphism $f: \pi \rightarrow Q$ such that the order of the kernel of $f$ is not divisible by $p$, and $Q$ satisfies the $p$-normalizer condition.

Proof. (i) and (ii) are obvious, since $\pi$ is $p$-normal, and the two normalizers coincide. For (iii) let $\pi_{p}$ be a $p$-Sylow subgroup and suppose that $c\left(\pi_{p}\right) \subseteq x \pi_{p} x^{-1}$ for some element $x$ belonging to $\pi$. Since $f\left(\pi_{p}\right)$ is a 
$p$-Sylow subgroup of $Q$ with centre $f\left(c\left(\pi_{p}\right)\right)$, the $p$-normality of $Q$ implies that $f\left(c\left(\pi_{p}\right)\right)=f(x) f\left(c\left(\pi_{p}\right)\right) f(x)^{-1}$. But $f \mid x \pi_{p} x^{-1}$ is a monomorphism, so that $c\left(\pi_{p}\right)=x c\left(\pi_{p}\right) x^{-1}$, showing that $\pi$ is $p$-normal. Since $f$ has a section, $f\left(N\left(\pi_{p}\right)\right.$ ) (respectively $f N\left(c \pi_{p}\right)$ ) is the normalizer of $f\left(\pi_{p}\right)$ (respectively of $c\left(f \pi_{p}\right)=g\left(c \pi_{p}\right)$ ). By the assumption on $Q$ the inclusion $f\left(N\left(\pi_{p}\right)\right) \subseteq f\left(N\left(c \pi_{p}\right)\right)$ is an $H_{*}\left(; \mathbb{F}_{p}\right)$ isomorphism, and since the kernel of $f$ has order prime to $p$, the same holds for $N\left(\pi_{p}\right) \subseteq N\left(c \pi_{p}\right)$. Therefore $\pi$ satisfies the $p$-normalizer condition.

On the other hand, the following criterion will also be useful.

LEMMA 1.5. Let $G$ be a finite group with $G_{2}$ a binary dihedral group of order larger than 8. If $H^{1}\left(G ; \mathbb{F}_{2}\right) \neq \mathbb{F}_{2} \oplus \mathbb{F}_{2}$ then $G$ does not satisfy the 2-normalizer condition.

Proof. The normalizer $N\left(G_{2}\right)$ acts on $H^{1}\left(G_{2} ; \mathbb{F}_{2}\right)=\mathbb{F}_{2} \oplus F_{2}$ by means of a map $N\left(G_{2}\right) \rightarrow$ Out $\left(G_{2}\right) \rightarrow G L\left(2, \mathbb{F}_{2}\right)$. Since $N\left(G_{2}\right) / G_{2}$ has odd order and the outer automorphism group of $G_{2}$ is a 2-group (cf. [Z]), the action of $N\left(G_{2}\right)$ on $H^{1}$ is trivial. Therefore, by Lemma $1.3, G$ satisfies the 2-normalizer condition only if $H^{1}\left(G ; \mathbb{F}_{2}\right)=\mathbb{F}_{2} \oplus \mathbb{F}_{2}$.

Refer now to the list of groups with periodic cohomology, given for example in [WF] or [TW]. There are six types to consider; four solvable and two non-solvable. Groups of Type I are metacyclic, and group of Types II, III, IV are split extensions

$$
N>\pi \stackrel{\leftrightarrow \rightarrow}{,},
$$

where $(|N|,|Q|)=1$, and $N$ is of Type $\mathrm{I}$. The quotient group $Q$ is isomorphic to a binary dihedral, generalized binary tetrahedral or generalized binary octahedral group, according to Type. We shall use the notation $D_{2 v}^{*}, T_{v}^{*}$ and $O_{v}^{*}$ for these groups (of order $2^{z}, 8 \cdot 3^{v}, 16 \cdot 3^{v}$ ), and appeal with little further comment to [WF] for facts about their structure. A group of Type $\mathrm{V}$ is a direct product $N \times S L\left(2, \mathbb{F}_{l}\right)$ with $l$ a prime $>3$, where $N$ is metacyclic and $\left(|N|, l\left(l^{2}-1\right)\right)=1$, and a group of Type VI is an extension of one of Type $\mathrm{V}$ by a cyclic group of order 2 .

THEOREM 1.6. Let $\pi$ be a finite group with periodic cohomology. Then

(i) $\pi$ satisfies the p-normalizer condition for all odd primes $p$.

(ii) $\pi$ satisfies the 2-normalizer condition if and only if:

(a) $\pi$ is of Type I, II or III, or

(b) $\pi$ is of Type $V$ and $l= \pm 3(\bmod 8)$.

Proof. (i) follows from Lemma 1.4(i). For the same reason groups of Type I satisfy the 2-normalizer condition. In case (iia) consider the split extension $N \hookrightarrow \pi \hookrightarrow Q$. If $Q$ is binary dihedral, i.e. $Q$ is embedded by a section as a 2-Sylow subgroup of $\pi$, the claim follows trivially from 
1.4(iii). If $Q$ is of tetrahedral type, then $Q$ order $8 \cdot 3^{v}(v \geqslant 1)$ and $N$ has order coprime with 6 . By 1.4 (iii) it is enough to verify the 2-normalizer condition for $T_{v}^{*}$. But this follows from 1.4(ii) since $T_{v}^{*}$ is itself a split extension of $D_{8}^{*}$ by a cyclic group $C_{3^{*}}$, i.e. the 2-Sylow subgroup is normal.

In case (iib) it is enough to check the 2-normalizer condition for $S L\left(2, \mathbb{F}_{l}\right)$. Note that the binary tetrahedral group

$$
T_{1}^{*}=\left\langle X, P, Q: X^{3}=1,\langle P, Q\rangle=D_{8}^{*}, X P X^{-1}=Q, X Q X^{-1}=P Q\right\rangle
$$

admits a faithful representation $\rho: T_{1}^{*} \rightarrow S L\left(2, \mathbb{F}_{l}\right)$ with image given by matrices $\left(\begin{array}{ll}a & b \\ c & d\end{array}\right)$ having entries equal to 0 or \pm 1 . The point of the restriction to $l \equiv \pm 3(\bmod 8)$ is that in this case a 2 -Sylow subgroup of $\rho T_{1}^{*}$ is also a 2-Sylow subgroup of $\operatorname{SL}\left(2, F_{l}\right)$. Indeed

$$
\left.\left(\rho T_{1}^{*}\right)_{1} \subseteq \rho T_{1}^{*} \subseteq N\left(\rho T_{1,2}^{*}\right)\right) \subseteq S L\left(2, \mathbb{F}_{l}\right) .
$$

Since a 2-Sylow subgroup of $S L\left(2, F_{l}\right)$ is of binary dihedral type, the 2-period equals 4 , see [S, thm. 1], and the Tate cohomology groups (with $F_{2}$ coefficients) are

$$
\hat{H}^{0}=\operatorname{Ker}\left(\mathbb{F}_{2} \stackrel{l\left(l^{2}-1\right)}{\longrightarrow} \mathbb{F}_{2}\right), \quad \hat{H}^{1}=\operatorname{Coker}\left(\mathbb{F}_{2} \stackrel{l\left(l^{2}-1\right)}{\longrightarrow} \mathbb{F}_{2}\right), \quad \hat{H}^{2}=\hat{H}^{3}=\{0\},
$$

since for $l>3, S L\left(2, \mathbb{F}_{1}\right)$ is perfect. The group $T_{1}^{*}$ is isomorphic to $S L\left(2, \mathbb{F}_{3}\right)$, hence the restriction map in $\hat{H}^{*}\left(\cdot, \mathbb{F}_{2}\right)$ from $S L\left(2, \mathbb{F}_{l}\right)$ to $\rho T_{1}^{*}$ is an isomorphism. Replacing Tate cohomology by ordinary homology the same follows for the two normalisers, and since by (1.1) $S L\left(2, \mathbb{F}_{l}\right)$ is 2-normal, the proof of the lemma is complete.

For the groups of Type IV we have $H^{1}\left(\pi ; \mathbb{F}_{2}\right)=\mathbb{F}_{2}$ since the commutator quotient of $O_{v}^{*}$ is cyclic of order two. Moreover, for the groups of Type $\mathrm{V}$ and $\mathrm{VI}, H^{1}\left(\pi ; \mathbb{F}_{2}\right)=\mathbb{F}_{2}$ or 0 , because $S L\left(2, \mathbb{F}_{l}\right)$ is perfect for $l$ a prime larger than 3 . Since all the groups $\pi$ not covered by (iia) or (iib) respectively, have $\pi_{2}$ equal to a binary dihedral group of order larger than 8 , we infer from (1.5) that they do not satisfy the 2-normalizer condition.

\section{Review of the localisation technique}

Let $K$ and $L$ denote arbitrary groups, and write $\operatorname{Hom}_{c}(K, L)$ for the set of conjugacy classes of homomorphisms $K \rightarrow L$. Thus

$$
\operatorname{Hom}_{c}(K, L) \cong[K(K, 1), K(L, 1)] \text {. }
$$


If $K$ is normal in $N$, there is an obvious action of $N$ on $\operatorname{Hom}_{c}(K, L)$ and $[K(K, 1), K(L, 1)]$, and we write

$$
\operatorname{Hom}_{c}(K, L)^{N} \cong[K(K, 1), K(L, 1)]^{N}
$$

for the stable elements with respect to these actions.

For any space $X$ we write $X_{Z / p}$ for the Bousfield $H_{*}(\cdot, Z / p)$ localization of $X$, see [B].

If $\pi$ is a finite group and $G$ a compact connected Lie group, there is a bijection

$$
[B \pi, B G] \cong \prod_{p|| \pi \mid}\left[(B \pi)_{Z / p}, B G\right] \text {. }
$$

This is obvious from the facts that $B G$ is 1 -connected, and the natural maps

$$
B \pi \rightarrow \prod_{p|| \pi \mid}(B \pi)_{Z / p} \leftarrow \bigvee_{p|| \pi \mid}(B \pi)_{Z / p}
$$

are $H_{*}(, Z)$-isomorphisms.

If as before $\pi$ is a finite group and $N_{\pi}\left(\pi_{p}\right)$ normalizes a $p$-Sylow subgroup, then by [WK1]

$$
\left[B N_{\pi}\left(\pi_{p}\right), X_{Z / p}\right] \cong\left[B \pi_{p}, X_{Z / p}\right]^{N_{\pi}\left(\pi_{p}\right)}
$$

for any 1-connected space $X$ of finite type. Taking $X=B G, G$ a compact connected Lie group then work of W. Dwyer, see [D], implies that

$$
\left[B \pi_{p}, B G\right] \cong \operatorname{Hom}_{c}\left(\pi_{p}, G\right) .
$$

Furthermore by naturality this isomorphism is compatible with the action by the normalizer. The next theorem, closely related to [WK2], is then an easy consequence.

THEOREM 2.5. If the finite group $\pi$ satisfies the p-normalizer condition, then for any compact connected Lie group $G$

$$
\left[(B \pi)_{z / p}, B G\right] \cong \operatorname{Hom}_{c}\left(\pi_{p}, G\right)^{N\left(\pi_{p}\right)} .
$$

Proof. Since $\pi$ satisfies the $p$-normalizer condition, the inclusion of $N\left(\pi_{p}\right)$ in $\pi$ induces an $H_{*}(; Z / p)$ isomorphism, compare Lemma 1.3 above. Hence

$$
\left[(B \pi)_{Z / p}, B G\right] \cong\left[(B \pi)_{Z / p},(B G)_{Z / p}\right] \cong\left[B N\left(\pi_{p}\right),(B G)_{Z / p}\right],
$$

and the result follows from (2.3) and (2.4).

If we combine (2.5) and Theorem 1.6 we obtain

THEOREM 2.6. Let $\pi$ be a finite group with periodic cohomology, satisfying the restrictions in (1.6a or b), and let $G$ be a compact, connected Lie group. Then

$$
[B \pi, B G] \cong \prod_{p|| \pi \mid} \operatorname{Hom}_{c}\left(\pi_{p}, G\right)^{N\left(\pi_{p}\right)} .
$$


In particular this result applies to the special linear groups $S L\left(2, F_{l}\right)$, $l \equiv \pm 3(\bmod 8)$. When $l=5$ we recover and improve the result of $\mathrm{J}$. F. Adams in $[\mathbf{A}]$ on the structure of $\left[B S L\left(2, \mathbb{F}_{5}\right), B S U(2)\right]$. This homotopy set is determined by its restrictions at the primes 2, 3 and 5; we obtain 12 homotopy classes, which can be described by means of virtual representations as follows:

$$
\left\{1, \psi^{k}\left(\iota_{ \pm}\right)(k=3,4,12), \psi^{l}\left(\iota_{+}\right)(l=5,15,20), \iota_{ \pm}\right\} .
$$

Here 1 corresponds to the trivial representation, $\iota_{ \pm}$to the two fixed point free representations of $S L\left(2, \mathbb{F}_{5}\right)$ in $S U(2)$ (the exceptional cuspidal representations in the language of Section 4 below), and $\psi^{k}$ denotes an unstable Adams operation. This example will be generalized in the final section.

\section{A special case, $G=S U(2)$}

Let $K$ and $L$ be arbitrary groups and let $f \in \operatorname{Hom}_{c}(K, L)$. If $x$ is an arbitrary element of a group $N$ containing $K$, define $f_{x} \in \operatorname{Hom}_{c}\left(x K x^{-1}, L\right)$ by

$$
f_{x}(y)=f\left(x^{-1} y x\right) \text { for all } y \in x K x^{-1} \text {. }
$$

By analogy with [S] we say that the map $f$ is stable (with respect to $N$ ) if for all $x \in N$

$$
f\left|K \cap x K x^{-1}=f_{x}\right| x K x^{-1} \cap K \text { as elements of } \operatorname{Hom}_{c}\left(K \cap x K x^{-1}, L\right) \text {. }
$$

Denote the set of $N$-stable elements in $\operatorname{Hom}_{c}(K, L)$ by $\operatorname{Hom}_{c}(K, L)^{N}$. If $K$ is a normal subgroup of $N$, then the stable elements are just those elements fixed under the induced $\mathrm{N}$-action (compare the previous section).

Since every map $g:(B \pi)_{Z / p} \rightarrow B G$ is of the form $f_{Z / p}$ for some map $f: B \pi \rightarrow B G$ (see (2.2)), it is clear that the set isomorphism in Theorem 2.5 factors through $\operatorname{Hom}_{c}\left(\pi_{p}, G\right)^{\pi}$. We state this as

LeмmA 3.1. Let $\pi$ and $G$ satisfy the assumptions of (2.5). Then

$$
\operatorname{Hom}_{c}\left(\pi_{p}, G\right)^{N\left(\pi_{p}\right)}=\operatorname{Hom}_{c}\left(\pi_{p}, G\right)^{\pi} \text {. }
$$

The conclusion of (3.1) holds only in very special circumstances, for example under the stringent assumptions in (2.5). The correct generalization of (2.6), in which we replace $N\left(\pi_{p}\right)$-invariant by the weaker condition $\pi$-stable and allow $\pi$ to be an arbitrary group with periodic cohomology, and which, as yet, we can only prove when $G=S U(2)$ is the following.

THEOREM 3.2. Let $\pi$ be a finite group with periodic cohomology. Then

(i) the natural map into complex $K$-theory

$$
[B \pi, B S U(2)] \rightarrow K(B \pi)=R(\pi)^{\wedge}
$$


is a monomorphism with image contained in the image of the completion map $R(\pi) \rightarrow R(\pi)^{\wedge}$ of the complex representation ring $R(\pi)$, and

$$
[B \pi, B S U(2)] \cong \prod_{p|| \pi \mid} \operatorname{Hom}_{c}\left(\pi_{p}, S U(2)\right)^{\pi} .
$$

Proof. Part (i) follows from (ii) and the diagram in the introduction since the completed complex representation ring $R(\pi)^{\wedge}$ is mapped $(1-1)$ into $\prod_{p|| \pi \mid} R\left(\pi_{p}\right)^{\wedge}$ by general properties of cohomology theories, and, since $\pi_{p}$ has prime power order, $\operatorname{Hom}_{c}\left(\pi_{p}, S U(2)\right)$ is mapped $(1-1)$ into $R\left(\pi_{p}\right)^{\wedge}$. Given the discussion in Section 2 and Lemma 3.1 the proof of (ii) is reduced to a problem at the prime 2 for those groups with periodic cohomology which do not satisfy the 2-normalizer condition. (We note that all groups in this class have 2-Sylow subgroups isomorphic to $D_{2}^{*}$ with $t \geqslant 4$.) It will now be enough to show that

$$
\left[(B \pi)_{z n}, B S U(2)\right] \cong \operatorname{Hom}_{c}\left(\pi_{2}, S U(2)\right)^{\pi}
$$

or equivalently, that

$$
\operatorname{Res}_{\pi \rightarrow \pi_{2}}:\left[B \pi, B S U(2)_{2}\right] \stackrel{\cong}{\rightrightarrows}\left[B \pi_{2}, B S U(2)_{2}^{\hat{2}}\right]^{\pi} .
$$

(The definition of the $\pi$-stable subset of the homotopy set on the right is entirely analogous to that of $\operatorname{Hom}_{c}\left(\pi_{2}, S U(2)\right)^{\pi}$.) The argument which we give below is motivated by and uses part of that in [M].

$\operatorname{Res}_{\pi \rightarrow \pi_{2}}$ is monic. Write $\left[B \pi, B S U(2)_{\hat{2}}\right]=F \cup U$, where $F$ consists of all elements which map to faithful homomorphisms in $\operatorname{Hom}_{c}\left(\pi_{2}, S U(2)\right)$, and $U$ the remainder. Theorem 1.7 from $[M]$ shows that $\operatorname{Res}_{\pi \rightarrow \pi_{2}} \mid F$ is monic when $\pi=S L\left(2, \mathbb{F}_{3}\right)$; in the proof one uses only the fact that the cohomology of this group is periodic at 2 . Hence the same argument applies in general.

Let $f$ belong to $U$, and write

$$
X_{\alpha}=\operatorname{map}\left(E \pi \underset{\pi}{\times} \pi / \pi_{\alpha}, B S U(2)_{2}\right){ }_{\rho_{\alpha}},
$$

where $\rho_{\alpha}: \pi_{\alpha} \rightarrow S U(2)$ is a homomorphism representing the restriction $f \mid B \pi_{\alpha}: B \pi_{\alpha} \rightarrow B S U(2) \hat{2}\left(\pi_{\alpha}\right.$ a 2-subgroup of $\pi$, compare the notation in [M]). Because $f$ is an element of $U$, and because the proper quotients of $D_{\mathcal{Z}^{\prime}}^{*}$, which are subgroups of $S U(2)$ are central $\left(C_{2}\right.$ and $\{1\}$ are the possibilities), we see that

$$
X_{\alpha} \simeq B S U(2)_{\hat{2}}
$$

This holds for each 2-subgroup $\pi_{\alpha}$ of $\pi$ (see also (3.1) in [M]). Hence the diagram of spaces $\left\{X_{\alpha}\right\}$, indexed as in [M] by $O_{2}(\pi)^{\text {op }}$, gives rise to a constant diagram $\left\{\pi_{i}\left(X_{a}\right)\right\}$ for each $i \geqslant 0$. It follows as in $[M]$ that $\operatorname{Res}_{\pi \rightarrow \pi_{2}} \mid U$ is monic. 
$\operatorname{Res}_{\pi \rightarrow \pi_{2}}$ is epic. Let $\varphi \in\left[B \pi_{2}, B S U(2)_{2}\right]^{\pi}$, which we identify with $\operatorname{Hom}_{c}\left(\pi_{2}, S U(2)\right)^{\pi}$. Hence $\varphi$ is represented by a map of the form $B \rho_{2}$ for some homomorphism $\rho_{2}: \pi_{2} \rightarrow S U(2)$. Once more it is useful to distinguish between the cases when $\rho_{2}$ is faithful and when $\rho_{2}$ factors through the centre of $S U(2)$. (The group $\pi_{2}$ is still isomorphic to $D_{2}^{*}$, $t \geqslant 4$.) If $\pi_{\alpha}$ is an arbitrary 2-subgroup of $\pi$, we obtain a well-defined class $B \rho_{\alpha}$ in $\left[B \pi_{\alpha}, B S U(2)\right]$ by first conjugating $\pi_{\alpha}$ onto a subgroup of $\pi_{2}$ and then restricting the homomorphism $\rho_{2}$. Note that in carrying out this step we use the $\pi$-stability of the class $\rho$. Again as in (3.3) we have a diagram of spaces indexed by $O_{2}(\pi)^{\text {op }}$. The homotopy inverse limit holim $X_{\alpha}$ is then non-empty if and only if there exists some $f$ in $\left[B \pi, B S U(2)_{2}\right]$ with $\operatorname{Res}_{\pi \rightarrow \pi_{2}}(f)=\varphi$ (once again see [M]). Since the spaces $X_{\alpha}$ are all simple, see [M, 3.1], we can again argue as in that paper to show that

$$
H_{0}\left(\operatorname{holim} X_{\alpha} ; F_{3}\right) \cong \mathbb{F}_{3} \text {. }
$$

This implies that the homotopy inverse limit is connected and non-empty.

\section{Examples}

(a) Let $D_{p q}$ be the non-abelian group of order $p q$, where $p$ and $q$ are distinct primes, $q<p$ and $q \mid p-1$. $D_{p q}$ has a presentation

$$
D_{p q}=\left\langle A, B: A^{p}=B^{q}=1, B A B^{-1}=A^{r}, r^{q} \bar{\equiv} 1(\bmod p)\right\rangle \text {. }
$$

The irreducible complex representations of $D_{p q}$ are of two kinds, one-dimensional of the form

$$
\beta_{h}\left\{\begin{array}{l}
A \mapsto 1 \\
B \mapsto \eta^{h}
\end{array},\right.
$$

and $q$-dimensional of the form

$$
\alpha_{k}\left\{\begin{array}{l}
A \mapsto \operatorname{diag}\left(\xi^{k}, \xi^{k r} \cdots \xi^{k r^{-1}}\right) \\
B \mapsto\left(\frac{0}{1} \mid \frac{1_{q-1}}{0}\right)
\end{array}\right.
$$

Here $\xi$ and $\eta$ are primitive $p$ th and $q$ th roots of unity $0 \leqslant h<q$, $1 \leqslant k<p$, and the second type of representation is obtained by transfer from the cyclic subgroup $C_{p}(A)$ generated by $A$. Since the defining extension is split, $D_{p q}$ retracts onto $C_{q}(B)$, and Theorem 2.6 shows that

$$
\begin{aligned}
{\left[B D_{p q}, B U(q)\right] \stackrel{\sim}{\rightrightarrows}\left[B C_{q}(B), B U(q)\right] \times\left[B C_{p}(A), B U(q)\right]^{B} } \\
=\operatorname{Hom}_{c}\left(C_{q}(B), U(q)\right) \times \operatorname{Hom}_{c}\left(C_{p}(A), U(q)\right)^{B} .
\end{aligned}
$$

As usual the superscript refers to those classes left invariant under the action of $B$. Since the structural map $C_{q}(B) \rightarrow$ Aut $\left(C_{p}(A)\right)$ is faithful, the 
invariant homomorphisms of $C_{p}(A)$ into $U(q)$ are represented on the generator $A$ by the matrix $\alpha_{k}(A)$ above. Calculating the characteristic roots shows that the periodic matrix $\alpha_{k}(B)$ is conjugate to $\operatorname{diag}\left\{1, \eta, \ldots, \eta^{q-1}\right\}$; as is well-known the single entry equal to 1 excludes the possibility of a free linear action on $S^{2 q-1}$. However if the class $f$ in $\left[B D_{p q}, B U(q)\right]$ restricts at the prime $q$ to a class represented by the sum

$$
\beta_{h_{1}}+\beta_{h_{2}}+\cdots+\beta_{h_{4}}
$$

then it is clear that $f$ is represented by the virtual representation

$$
\epsilon \alpha_{\kappa}+\sum_{1} \beta_{h(1)}-\sum_{2} \beta_{h(2)}
$$

where either $\epsilon=0, \sum_{1}$ the sum (4.2) and $\sum_{2}=0$, or $\epsilon=1$, the first sum running over those values of $h_{l}$ not occurring in $\alpha_{k} \mid C_{q}(B)=$ $\sum_{j=0}^{q-1} \beta_{j} \mid C_{q}(B)$, and the second sum removing those summands not required. Note also that the class of $k \bmod p$ is uniquely determined up to a power of $r$ by the restriction of $f$ to $B C_{p}(A)$; there are therefore $q^{q-1}(p+q-1)$ possibilities for the homotopy class $f$. In particular, one can manufacture a class $f$ whose Euler class $e(f)$ generates $H^{2 q}\left(D_{p q}, Z\right)=Z / p q Z$, for example

$$
f=\alpha_{k}+\beta_{1}-(1) \text {. }
$$

The positive representation $\alpha_{k}+\beta_{1}$ in (4.4) is the one used in $[P]$ to construct a free action by $D_{p q}$ on a Brieskorn variety, which is then replaced using surgery in the middle dimension by a sphere, at least when $p>q \geqslant 3$. (The negative summand $-(1)=-\beta_{0}$ in $f$ can be thought of corresponding to a normal bundle.)

With suitable complications to the arithmetic it is clear that this discussion extends to other metacyclic groups of Type I. As a first step replace $D_{p q}$ by $D_{p q^{2}}^{*}$, an extension of $C_{p}(A)$ by $C_{q^{2}}(B)$ in which the structural map

$$
C_{q^{2}}(B) \rightarrow \text { Aut }\left(C_{p}(A)\right)
$$

has kernel of order $q$. (We may think of this group as an odd order variant of the binary dihedral group.) In this case one obtains $q$ dimensional representations $\alpha_{k, l}$ by transfer from the normal cyclic subgroup generated by $A B^{q}$, which do define free linear actions on $S^{2 q-1}$. The difference from the $p q$-case is explained by the replacement of the entry 1 by $\eta^{\prime}$ in the lower left-hand corner of the matrix $\alpha_{k, l}(B)$. However, by "mixing" representations as in (4.3) above it is possible to find classes $\alpha_{k, 1}+\beta_{h}-\beta_{h}$, which correspond to homotopically exotic 
free actions. Again restricting attention to the case $p>q \geqslant 3$ there is no reason to suppose that an argument similar to Petrie's in [P] fails, which implies that it is possible to choose the $k$-invariant of a free topological action on $S^{2 q-1}$ to restrict to any given generator of $H^{2 q}\left(C_{q^{2}}(B), Z\right)$. If this heuristic argument is correct, we have recovered Martin Taylor's description of the Swan subgroup for $D_{p q}^{*}$. (This remains unpublished, but uses methods of $\mathbf{A}$. Fröhlich, see $[\mathbf{F}]$.)

(b) Let $\pi=S L\left(2, F_{l}\right), l \equiv 5(\bmod 8)$, so that (i) a 2-Sylow subgroup $\pi_{2}$ has order 8 and Theorem 2.6 applies, and (ii) the cohomological period equals $l-1$ (compare [S]). Furthermore (iii) there exist free smooth actions by $\pi$ on $S^{l-2}$ which may be taken to be linear only if $l=5$, see [MTW]. With the previous example as motivation we consider the set isomorphism defined by restriction

$$
\left[B \pi, B U\left(\frac{l-1}{2}\right)\right] \cong \prod_{p \mid l\left(l^{2}-1\right)}\left[B \pi_{p}, B U\left(\frac{l-1}{2}\right)\right]^{\pi} .
$$

We need the following information about $S L\left(2, \mathbb{F}_{l}\right)$, see [DF] Vol. 1, Ch. 38, for more detail. Let $\mu$ generate $\mathbb{F}_{l}$ as an additive group, and $v$ generate the cyclic group $\mathbb{F}_{l}^{x}=\mathbb{F}_{l}-\{0\}$ of order $l-1$. Let

$$
D=\left(\begin{array}{cc}
v & 0 \\
0 & v-1
\end{array}\right), \quad A=\left(\begin{array}{ll}
1 & 0 \\
\mu & 1
\end{array}\right)
$$

and $B$ an element of order $l+1$, which diagonalizes over the field $\mathbb{F}_{l^{2}} . A$ and $D$ generate a subgroup $K$ of index $l+1$, which is actually the semi-direct product $F_{l} \bar{x} \mathbb{F}_{1}^{x}$ in which $v$ acts on $\mu$ by the square of the usual action. We obtain one family of complex representations $x_{k}$ by transfer from this subgroup; for $1 \leqslant k \leqslant\left(\frac{l-3}{2}\right)$ these are irreducible and the last one splits. The second family $\Theta_{h}$ of cuspidal representations is harder to describe; for $1 \leqslant h \leqslant\left(\frac{l-1}{2}\right)$ there are again irreducible, but $\boldsymbol{\Theta}_{(1+1) / 2}$ splits as the sum two exceptional representations $\gamma_{1}$ and $\gamma_{2}$ both of degree $\left(\frac{l-1}{2}\right)$. Besides these two families there is the trivial representation of degree 1 and a final irreducible representation of degree $l$. Of particular interest to us are the following virtual representations (of Brạuer type):

$$
\beta_{k, h}=x_{k}-\Theta_{h}
$$

If $\delta, \xi$ and $\eta$ are $r$ th primitive roots of unity for $r=l-1, l, l+1$, then the character of $\beta_{k, h}$ is as follows:

$$
D \mapsto \delta^{k}+\bar{\delta}^{k}, \quad A \mapsto 2 \text { and } B \mapsto \eta^{h}+\bar{\eta}^{h} .
$$


These virtual representations are all of the form $\psi^{j} \beta_{1,1}$ for suitable $j$ 's; we will see below that the set

$$
\left\{\psi^{j} \beta_{1,1} \mid j \geqslant 0\right\}
$$

suffices to describe the restriction of $\left[B \pi, B U\left(\frac{l-1}{2}\right)\right]$ away from the prime $l$. However in order to handle $l$ we need the two exceptional cuspidal representations $\left(\delta_{i}, i=1,2\right)$ with characters:

$$
\begin{aligned}
& D \mapsto 0, \quad A \mapsto \frac{1}{2}(-1 \pm \sqrt{ } l) \\
&\quad \text { (depending on whether } i=1 \text { or } 2) \text { and } B \mapsto 1 .
\end{aligned}
$$

Since we assume that $l-1$ is divisible by 4 we have $+l$ rather than $-l$ under the root sign, see [DF] page 228 for the complete character table.

In looking more closely at the decomposition of $\left[B \pi, B U\left(\frac{l-1}{2}\right)\right]$ in (4.5) it is clear that away from the prime 2 we need only consider the stable subsets of

$$
\left[B \pi_{1}, B U\left(\frac{l-1}{2}\right)\right]\left[B \pi_{l-1}, B U\left(\frac{l-1}{2}\right)\right] \text { and }\left[B \pi_{l+1}, B U\left(\frac{l-1}{2}\right)\right]
$$

where $\pi_{l}=C_{l}(A)$ and, by abuse of notation, we write $\pi_{l-1}$ (respectively $\left.\pi_{l+1}\right)$ for $C_{l-1}(D)$ (respectively $C_{l+1}(B)$ ). If we recall the action of $\mathbb{F}_{l}^{\times}$on $\mathbb{F}_{l}$ which defined the (Borel) subgroup $K$, namely $\mu \mapsto v^{2} \mu$, it is clear that at the prime $l$ the stable elements are picked up by $\operatorname{Res}_{\pi \rightarrow \pi_{l}}\left(\gamma_{t}\right)$ for $i=1,2$. The stable part of $\left[B \pi_{l-1}, B U\left(\frac{l-1}{2}\right)\right]$ equals the invariant part under conjugation by elements of the normalizer $N\left(\pi_{l-1}\right)$, compare Lemma 3.1, which is actually a group of quaternion type acting at worst by inversion. Hence the image under restriction consists of classes which decompose as $\left(\frac{l-1}{4}\right)$ summands of the form $\delta^{k}+\delta^{k}$. The situation at $(l+1)$ is similar; we obtain $\left(\frac{l-1}{4}\right)$ summands of the form $\eta^{h}+\bar{\eta}^{h}$. It is clear from the defining equation (4.6) that the virtual representations $\beta_{k, h}$ and $\psi^{l+1} \beta_{k, h}$ cover both images. Finally there is no problem at the prime 2. Stability ensures that apart from the trivial representation, we are only concerned with sums of copies of the unique faithful representation of $D_{8}^{*}$ in SU(2); this can be seen from the natural bijection

$$
\left[B D_{8}^{*}, B S^{1}\right]^{\pi} \cong H^{1}\left(B \pi ; Z_{(2)}\right)=0 .
$$

Because $\beta_{1,1}$ restrict on $D_{8}^{*} \subseteq \pi$ to the unique faithhful irreducible representation, we conclude finally that the elements of $\left[B \pi, B U\left(\frac{l-1}{2}\right)\right]$ 
correspond to (equivalence classes) of virtual representations of the form

$$
\epsilon_{1} \gamma_{1}+\epsilon_{2} \gamma_{2}+\sum_{1} \psi^{j(1)} \beta_{1,1}-\sum_{2} \psi^{j(2)} \beta_{1,1}
$$

of formal dimension $\left(\frac{l-1}{2}\right)$, with $\epsilon_{i}=0$ or 1 , and $\epsilon_{1} \epsilon_{2} \neq 1$.

Remark. It is clear that, as in the case of metacyclic groups, it is possible to choose a class $f \in\left[B S L\left(2, \mathbb{F}_{l}\right), B U\left(\frac{l-1}{2}\right)\right]$, given in terms of a virtual representation $\rho$ and with top dimensional Chern class generating the cyclic group $H^{l-1}\left(S L\left(2, \mathbb{F}_{l}\right), Z\right)$; for instance, one easily checks using the character table (loc. cit.) that $\gamma_{1}+\beta_{1,1}-\beta_{(l-1) / 4,1}$ restricts on every Sylow subgroup of $\pi$ to a fixed point free representation, giving therefore rise to a $\rho$ of the type desired. It would be most interesting to know if $\rho$ can be used to construct a free action by the group on some generalized Brieskorn variety. Because of the number of homomorphisms making up $\rho$ we do not expect this to be easy in general.

\section{REFERENCES}

A. J. F. Adams, 'Maps between classifying spaces I', Inventiones Math. 49 (1978) $1-65$.

B. A. K. Bousfield, 'The localization of a space with respect to homology', Topology 14 (1975), 133-150.

D. W. G. Dwyer, 'Maps between classifying spaces', (preprint 1986).

DF. L. Dornhoff, Group representation theory, Marcel Dekker (New York) 1972.

F. A. Fröhlich, 'Arithmetic and Galois module structure for tame extensions', $J$. für reine und angew. Mathematik 286/7 (1976) 380-440.

I. K. Ishiguro, 'Unstable Operations on classifying spaces', (preprint 1986).

M. G. Mislin, 'The homotopy classification of self-maps of infinite quaternionic projective space', Quart. J. Math. Oxford (2), 38 (1987), 245-257.

MTW. I. Madsen, C. B. Thomas and C. T. C. Wall, 'Topological spherical space form problem III', Pacific J. Math. 106 (1983) 135-143.

P. T. Petric, 'Free metacyclic group actions on homotopy spheres', Annals of Math. 94 (1971) 108-124.

S. R. G. Swan, 'The period of a finite group', Illinois J. Math., 4 (1960) 341-346.

T1. C. B. Thomas, 'Free actions by finite groups on $S^{3}$, $A M S$ Symposia 32 (1978) 125-130.

T2. C. B. Thomas, 'A reduction theorem for free actions by $Q(8 \pi ; k, l)$ ', Bull. London Math. Soc. (to appear).

TW. C. B. Thomas and C. T. C. Wall, 'The topological spherical space form problem I', Compositio Mathematica 23 (1) 1971, 101-114.

WF. J. Wolf, Spaces of constant curvature, nth. edition (Publish or Perish Inc.)

WK1. Z, Wojtkowiak, 'When a map equivariant up to homotopy is homotopic to an equivariant one' (preprint 1986). 
WK2. Z. Wojtkowiak, 'Some remarks on maps from $B \pi$ into $X^{\prime}$ (preprint 1986).

Z. H. Zassenhaus, The theory of groups, Chelsea (New York) 1949 or 1958.

ETH Mathematik,

ETH-Zentrum, CH-8092 Zurich, Switzerland 